\title{
In vivo tumor targeting and imaging with anti-vascular endothelial growth factor antibody-conjugated dextran-coated iron oxide nanoparticles
}

\author{
This article was published in the following Dove Press journal: \\ International Journal of Nanomedicine \\ 6 June 2012 \\ Number of times this article has been viewed
}

\section{Wan-Ju Hsieh' \\ Chan-Jung Liang' \\ Jen-Jie Chieh ${ }^{4}$ \\ Shu-Huei Wang' \\ I-Rue Lai' \\ Jyh-Horng Chen ${ }^{2}$ \\ Fu-Hsiung Chang ${ }^{3}$ \\ Wei-Kung Tseng ${ }^{4-6}$ \\ Shieh-Yueh Yang ${ }^{4}$ \\ Chau-Chung Wu ${ }^{7}$ \\ Yuh-Lien Chen' \\ 'Institute of Anatomy and Cell Biology, \\ College of Medicine, ${ }^{2}$ Department \\ of Electrical Engineering, ${ }^{3}$ Institute \\ of Biochemistry and Molecular Biology, \\ National Taiwan University, Taipei, Taiwan; \\ ${ }^{4}$ Institute of Electro-Optical Science and \\ Technology, National Taiwan Normal \\ University, Taipei, Taiwan; ${ }^{5}$ Division of \\ Cardiology, Department of Internal Medicine, \\ E-Da Hospital, Taipei, Taiwan; ${ }^{6}$ Department \\ of Medical Imaging and Radiological Sciences, \\ I-Shou University, Taipei, Taiwan; ${ }^{7}$ Department \\ of Internal Medicine and Primary Care \\ Medicine, National Taiwan University \\ Hospital, Taipei, Taiwan}

Correspondence: Yuh-Lien Chen

Department of Anatomy and Cell Biology,

College of Medicine, National Taiwan

University, No I, Section I, Ren-Ai Rd,

Taipei, 100, Taiwan

Tel +88 $622312345688 \mid 76$

Fax +88 $623393 \quad 1713$

Email ylchenv@ntu.edu.tw

Chau-Chung Wu

Department of Internal Medicine and

Primary Care Medicine, National Taiwan

University Hospital, 7 Chung-Shan S Rd,

Taipei, Taiwan

$\mathrm{Tel}+88622312345665408$

Fax +886223914853

Email chauchungwu@ntu.edu.tw
Background: Active targeting by specific antibodies combined with nanoparticles is a promising technology for cancer imaging and detection by magnetic resonance imaging (MRI). The aim of the present study is to investigate whether the systemic delivery of antivascular endothelial growth factor antibodies conjugating to the surface of functionalized supermagnetic iron oxide nanoparticles (anti-VEGF-NPs) led to target-specific accumulation in the tumor.

Methods: The VEGF expression in human colon cancer and in Balb/c mice bearing colon cancers was examined by immunohistochemistry. The distribution of these anti-VEGF-NPs particles or NPs particles were evaluated by MRI at days 1, 2, or 9 after the injection into the jugular vein of Balb/c mice bearing colon cancers. Tumor and normal tissues (liver, spleen, lung, and kidney) were collected and were examined by Prussian blue staining to determine the presence and distribution of NPs in the tissue sections.

Results: VEGF is highly expressed in human and mouse colon cancer tissues. MRI showed significant changes in the $\mathrm{T}^{*}{ }_{2}$ signal and $\mathrm{T}_{2}$ relaxation in the anti-VEGF-NP- injected-mice, but not in mice injected with NP alone. Examination of paraffin sections of tumor tissues stained for the iron constituent of the NPs with Prussian blue revealed a strong blue reaction in the tumors of anti-VEGF-NP-treated mice, but only a weak reaction in mice injected with NPs. In both groups, at all time points, Prussian blue-stained liver and spleen sections showed only light staining, while stained cells were rarely detected in kidney and lung sections. Transmission electron microscopy showed that many more electron-dense particles were present in endothelial cells, tumor cells, and extracellular matrix in tumor tissues in mice injected with anti-VEGFNPs than in NP-injected mice.

Conclusion: These results demonstrated in vivo tumor targeting and efficient accumulation of anti-VEGF-NPs in tumor tissues after systemic delivery in a colon cancer model, showing that anti-VEGF-NPs have potential for use as a molecular-targeted tumor imaging agent in vivo.

Keywords: nanoparticles, vascular endothelial growth factor, colon tumor, magnetic resonance imaging, transmission electron microscopy

\section{Introduction}

Cancer remains the leading cause of death in the world and colorectal cancer accounts for nearly half of the annual cancer deaths, with nearly 1.2 million new cases of colon cancers being diagnosed each year. ${ }^{1}$ Despite advances in the understanding of the pathogenesis, the discovery of cancer biomarkers, and improvements in surgery, radiotherapy, and chemotherapy, the overall survival 
rate from cancer has not significantly improved in the past two decades. ${ }^{2}$ Novel tools or agents for early detection and diagnosis of cancers are therefore urgently required to increase patient survival. Magnetic resonance imaging (MRI) provides superb image resolution and is often used as a tool for cancer detection. Gadolinium diethylenetriaminopentaacetic acid is often used as the magnetic contrast agent to obtain contrast enhancement and signal amplification during MRI, but has a very short blood retention time, low bioavailability, and high toxicity. ${ }^{3}$ Recently, supermagnetic iron oxide nanoparticles (NPs) lacking these three drawbacks became available as novel diagnostic tools for noninvasively assessing the benefit of therapies, identifying early molecular changes and reoccurrence associated with cancer, and are being used in new targeted and specific therapies. ${ }^{4}$

Tumor growth and metastatic dissemination largely depend on new blood vessel formation (angiogenesis), and vascular endothelial growth factor (VEGF) is a key regulator of this process. ${ }^{6} \mathrm{VEGF}$ is an endothelial cell-specific mitogen used in vitro, and an angiogenic inducer in a variety of in vivo contexts, ${ }^{6}$ and is also a vascular permeability factor. $^{7}$ In situ hybridization studies have demonstrated high VEGF mRNA levels in the majority of human tumors. ${ }^{8-11}$ Moreover, VEGF protein expression is not restricted to the advanced stages of colonic cancer, but can also be observed in the premalignant stages of tumor development when compared to normal colonic mucosa. ${ }^{12}$ As VEGF expression is localized in the tumor, and VEGF is expressed during the pathogenesis of cancer, this provides an opportunity for designing VEGF-targeted approaches for cancer detection and treatment. ${ }^{13,14}$ Active targeting by specific antibodies combined with nanosize superparamagnetic iron oxide is a promising technology for MRI technology. ${ }^{15}$ In this study, we combined an anti-VEGF antibody as a tumor-targeting ligand and dextran-coated $\mathrm{Fe}_{3} \mathrm{O}_{4} \mathrm{NPs}$ to generate anti-VEGF antibody-conjugated dextran-coated $\mathrm{Fe}_{3} \mathrm{O}_{4}$ nanoparticles (anti-VEGF-NPs) that selectively accumulated in the tumor of CT26 tumor-bearing mice in vivo with a high efficiency, allowing the location of the colon tumors to be delineated by MRI. The anti-VEGF-NPs specifically bound to, and were internalized by, VEGF-expressing endothelial cells and tumor cells, as shown by Prussian blue staining and transmission electron microscopy (TEM). Our results show that anti-VEGF-NPs can be used as an antibody-targeted MRI contrast agent for the in vivo detection of VEGF-expressing tumors.

\section{Materials and methods} Synthesis of dextran-coated $\mathrm{Fe}_{3} \mathrm{O}_{4}$ nanoparticles (NP) and anti-VEGF antibody-conjugated dextran-coated $\mathrm{Fe}_{3} \mathrm{O}_{4}$ nanoparticles (anti-VEGF-NP)

The protocol for the synthesis of the magnetic $\mathrm{Fe}_{3} \mathrm{O}_{4}$ nanoparticles was proposed by MagQu Co, Ltd (Taipei, Taiwan) and has been described previously. ${ }^{16}$ Briefly, a ferrite solution containing a stoichiometric 1:2 ratio of ferrous sulfate heptahydrate $\left(\mathrm{FeSO}_{4} \cdot 7 \mathrm{H}_{2} \mathrm{O}\right)$ and ferric chloride hexahydrate $\left(\mathrm{FeCl}_{3} \cdot 6 \mathrm{H}_{2} \mathrm{O}\right)$ was mixed with an equal volume of aqueous dextran, which acts as a surfactant for $\mathrm{Fe}_{3} \mathrm{O}_{4}$ particles dispersed in water. The mixture was heated to $70^{\circ} \mathrm{C}-90^{\circ} \mathrm{C}$ and titrated with a strong base solution to form black $\mathrm{Fe}_{3} \mathrm{O}_{4}$ particles.

Aggregates and excess unbound dextran were removed by centrifugation and gel filtration chromatography to obtain a highly concentrated magnetic fluid containing dextran-coated $\mathrm{Fe}_{3} \mathrm{O}_{4}$ NPs. The desired concentration of the magnetic reagent was produced by diluting the highly concentrated magnetic fluid with phosphate-buffered saline (PBS; pH 7.4). To bind the anti-VEGF Abs (sc507; Santa Cruz Biotechnology, Santa Cruz, CA) to the dextran on the outer shell of the magnetic nanoparticles, $\mathrm{NaIO}_{4}$ solution was added to the magnetic solution to oxide dextran and create aldehyde groups, which then reacted with amino groups on the antiVEGF antibody, resulting in covalent attachment. Magnetic separation was then used to separate the anti-VEGF-NPs (MF-VEG-0060, MagQu) from nonbound anti-VEGF antibody. The concentration of the magnetic reagent in terms of magnetism was $0.3 \mathrm{emu} / \mathrm{g}(\sim 3.6 \mathrm{mg}-\mathrm{Fe} / \mathrm{mL})$. The sizes of NPs and anti-VEGF-NPs were measured with two different methods, dynamic light scattering (DLS) and transmission electron microscopy (TEM). In the DLS assay, the samples were diluted in double-distilled water and examined using a Nanotrac-150 (Microtrac Inc, Montgomeryville PA). In TEM observation, the particles were negatively stained with sodium phosphotungstate solution.

\section{Cancer cell line}

CT26 cells, a murine colon adenocarcinoma cell line (ATCC, Manassas, VA), were grown in Roswell Park Memorial Institute medium containing penicillin-streptomycin (1\%) and fetal bovine serum (FBS, 10\%; Biological Industries Ltd, Beit Ahemeq, Israel) at $37^{\circ} \mathrm{C}$ in a humidified atmosphere of $95 \%$ air and $5 \% \mathrm{CO}_{2}$. 


\section{Establishment of the colon tumor model}

Forty-eight Balb/c mice of either sex (6 to 8 weeks of age) were purchased from BioLASCO (Taipei, Taiwan). All procedures involving experimental animals were performed in accordance with the guidelines for animal care of the National Taiwan University and complied with the Guide for the Care and Use of Laboratory Animals. Colon tumors were generated in mice by subcutaneous implantation of $5 \times 10^{5}$ CT26 cells suspended in $150 \mu \mathrm{L}$ of Roswell Park Memorial Institute medium. After injection, the animals were given food and water ad libitum, and were housed under normal conditions with a 12-hour light/dark cycle and were used for experiments after 14 days.

\section{MRI scan in vivo}

On day 14 after tumor injection, images were obtained under isoflurane anesthesia ( $1 \%$ to $2 \%$ isoflurane; $1 \mathrm{~L} \mathrm{O}_{2}$ ) at the $7 \mathrm{~T}$ animal MRI Core Laboratory of the Neurobiology and Cognitive Science Center (National Taiwan University, Taipei). To follow MRI contrast changes, the mice were then injected through the jugular vein with anti-VEGF-NPs or NPs $(10 \mathrm{mg} /$ $\mathrm{Kg}$ of body weight) in $100 \mu \mathrm{L}$ of PBS and imaged at different time points ( 1 day, 2 days, and 9 days) according to the previous studies. ${ }^{17,18}$ Blood pressure and heart rate were measured before and after injection. Images were taken pre- and post-injection to evaluate the contrast enhancement using the target-specific contrast agent. For MRI images, a 7-Tesla MRI system (Burker BioSpin Biospec 7030, Ettlingen, Germany) with a $30 \mathrm{~cm}$ horizontal bore, a 75 Gauss/cm gradient, and a $35 \mathrm{~mm}$ transmit-receive birdcage volume coil was used. Axial images over the flank were acquired with a $2.0 \mathrm{~cm}$ square field of view and $1.0 \mathrm{~mm}$ slice thickness. MRI was performed using a T 2 -weighted gradient echo pulse sequence (repetition time/echo time 244/4.3 milliseconds, number of excitations 8 , matrix $=256 \times 256$, resolution $78 \times 78 \times 1000 \mu \mathrm{m}$ ). The signal of the phantom was used to normalize the signals.

\section{Preparation of cell and tissue lysates and Western blot analysis}

Western blot analyses were performed as described previously. ${ }^{19}$ To prepare cell lysates, the cells or tissue were lysed for 1 hour at $4^{\circ} \mathrm{C}$ in lysis buffer $(20 \mathrm{mM}$ Tris- $\mathrm{HCl}, 150 \mathrm{mM}$ $\mathrm{NaCl}, 1 \mathrm{mM}$ EDTA, 1 mM EGTA, 1\% Triton X-100, 1 mM phenylmethylsulfonyl fluoride, $\mathrm{pH}$ 7.4). The lysate was then centrifuged at $4000 \mathrm{~g}$ for $30 \mathrm{~min}$ at $4^{\circ} \mathrm{C}$, and the supernatant retained. Samples of cell lysate ( $25 \mu \mathrm{g}$ of protein) were subjected to $15 \%$ sodium dodecyl sulfate polyacrylamide gel electrophoresis and transferred to polyvinylidene fluoride membranes, which were then treated for 1 hour at room temperature with $3 \%$ nonfat milk in Tris-buffered saline (50 mM TrisHCl, pH 7.4, $150 \mathrm{mM} \mathrm{NaC}$ ) containing $0.1 \%$ Tween 20 (TBST) to block nonspecific binding of antibodies. The membranes were then incubated overnight at $4^{\circ} \mathrm{C}$ with rabbit antibodies against human VEGF (1:1000; Santa Cruz Biotechnology) diluted in Tris-buffered saline with Tween containing $0.1 \%$ bovine serum albumin, then again for 1 hour at room temperature with horseradish peroxidaseconjugated goat anti-rabbit immunoglobulin-G antibodies (1:2000 in Tris-buffered saline with Tween, $0.1 \%$ bovine serum albumin; Santa Cruz Biotechnology), which are bound antibodies being detected using Chemiluminescence Reagent Plus (NEN, Boston, MA). The intensity of each band was quantified using a densitometer. Antibodies against glyceraldehyde-3-phosphate dehydrogenase (1:5000; Santa Cruz Biotechnology) were used as loading controls.

\section{Immunohistochemical procedures}

Immunohistochemistry was used to evaluate the VEGF expression in both human colon tumors and a mouse model of colon tumor and to compare the difference of its expression between tumor tissues and normal tissues. Human tumor samples were processed with the approval of the National Taiwan University Hospital and Research Ethics Boards. Human and mouse tumor tissues and normal mouse organs (liver, kidney, lung, and spleen) were fixed in 4\% paraformaldehyde, embedded in paraffin, and sectioned for detection of VEGF expression using immunohistochemical staining. The first of two serial sections was stained overnight at $4^{\circ} \mathrm{C}$ with rabbit anti-VEGF antibody (1:150 dilution in PBS; Santa Cruz Biotechnology), washed twice with PBS, and then incubated for 1 hour at $37^{\circ} \mathrm{C}$ with fluorescein isothiocyanateconjugated goat anti-rabbit immunoglobulin-G antibodies (1:200 dilution in PBS; Santa Cruz Biotechnology), and observed under the fluorescence microscope. The second serial section was stained with hematoxylin-eosin staining.

\section{Histological analysis by Prussian blue staining}

Mice were sacrificed at days 1, 2, or 9 after injection of anti-VEGF-NPs or NPs, and tumor and normal tissues (liver, spleen, lung, and kidney) were collected. Paraffin tissue sections were examined by Prussian blue staining to determine the presence and distribution of NPs in the tissue sections. The paraffin sections were incubated for $30 \mathrm{~min}$ at 
room temperature with staining solution (a 1:1 mixture of $4 \%$ potassium ferrocyanide and $4 \%$ hydrochloric acid). After counterstaining with $0.5 \%$ neutral red solution, the slides were then examined under a light microscope.

\section{TEM}

To examine the detailed distribution of anti-VEGF-NPs and NPs, TEM was performed on tumor samples taken 1 day after nanoparticle injection. Tumor samples were postfixed for 1 hour with $1 \%$ osmium tetroxide in $0.1 \mathrm{M}$ phosphate buffer, dehydrated in a graded series of ethanols, and embedded in Epon. Thin sections were picked up on 150-mesh copper grids, lightly stained with lead citrate, and scanned in a Hitachi H7100 (Hitachi, Tokyo, Japan) at $100 \mathrm{kV}$, with high-resolution digital images being obtained using an AMT digital CCD camera.

\section{Statistical analysis of data}

All values are presented as the mean \pm SEM and were analyzed using Student's $t$-test. Statistical significance was determined as $P<0.05$.

\section{Results \\ VEGF is highly expressed in human and mouse colon cancer tissues}

VEGF expression in human and mouse colon tissue was evaluated by immunofluorescent staining. In surgically removed normal tissue and tumor tissue in human colon tumor samples, VEGF immunoreactivity was strong in the tumor tissue, but undetectable in the normal tissue (Figure 1A). Colon tumors were easily distinguishable from the healthy colon when examined and visualized by hematoxylin-eosin staining. In tumor sections from CT26-bearing mice, strong VEGF immunoreactivity was detected specifically in the tumors (Figure 1B). VEGF expression was weak in the spleen and undetectable in the lung, liver, and kidney (Figure 1B). To confirm the level of expression of VEGF in vitro and in vivo, Western blotting was used. As shown in Figure 1C, high VEGF expression was detected not only in cultured CT26 cells, but also in cancer tissue in the mouse model, while VEGF expression was barely detectable in the spleen and undetectable in the lung, liver, and kidney. These results

A

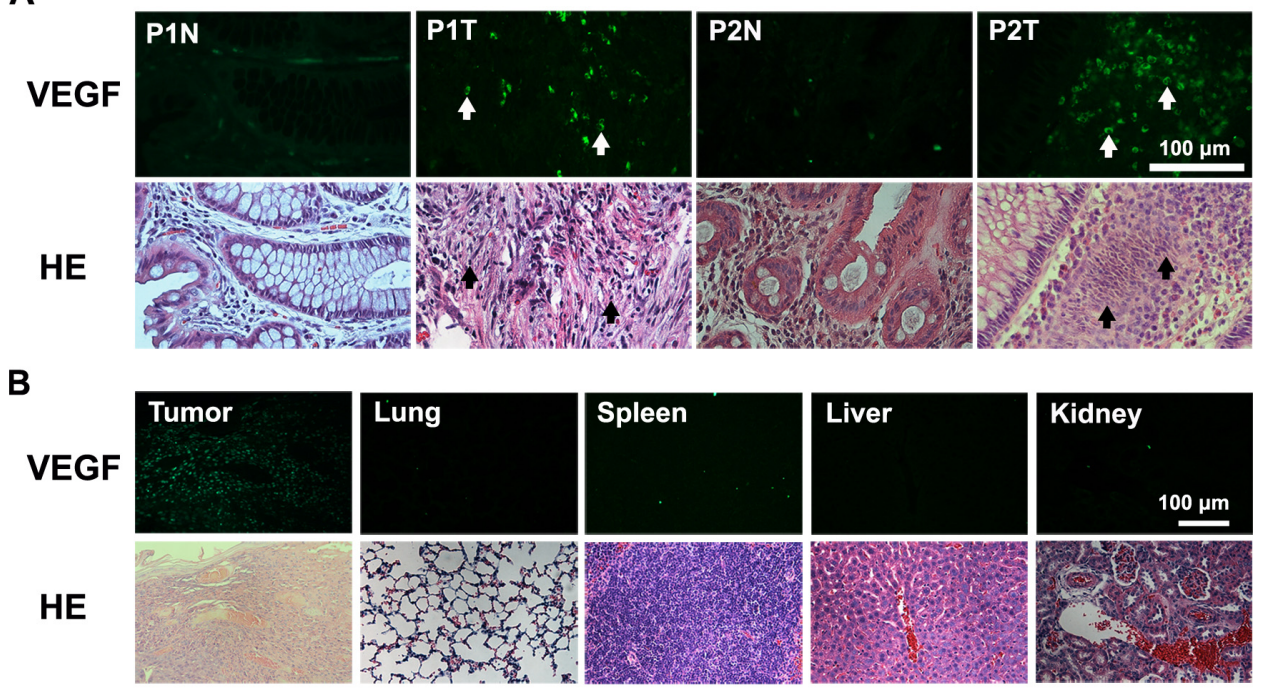

C

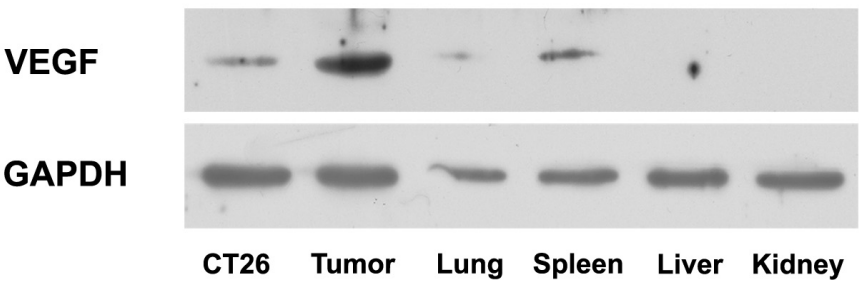

Figure I Strong expression of VEGF in human and mouse colon tumors as shown by immunofluorescent staining and Western blotting. (A) Serial sections from $\mathrm{N}$ and $\mathrm{T}$ in colon tumor specimens from PI and P2 were stained for VEGF (upper panels) or with HE staining (lower panels). The arrows indicate VEGF expression colocalized with tumor cells. Scale bar $=100 \mu \mathrm{m}$. (B) Fluorescence and light microscopic images of mouse tumor sections. Serial sections from tumor, lung, spleen, liver, and kidney of CT26bearing mice were stained for VEGF (upper panels) or HE (lower panel). Scale bar = $100 \mu \mathrm{m}$. (C) VEGF levels in CT26 cell lysates and in tissue lysates from tumor, lung, spleen, liver, and kidney of CT26-bearing mice measured by Western blotting. GAPDH was used as the loading control.

Abbreviations: GAPDH, glyceraldehyde-3-phosphate dehyodrogenase; HE, hematoxylin and eosin; N, normal tissue; P, patient; T, tumor tissue; VEGF, vascular endothelial growth factor. 
indicate that VEGF is highly expressed in tumor cells and that the anti-VEGF antibody bound to its target, VEGF, in both mouse and human tumor tissue sections, making this molecule as an ideal target for positive selection of VEGFtargeted imaging agents. In addition, this mouse model was suitable for in vivo assessment of tumor targeting/imaging using anti-VEGF antibody.

\section{Anti-VEGF-NPs, but not NPs, specifically target colon cancer in vivo as shown by MRI}

To permit evaluation of VEGF expression using light microscopy or MRI and to develop a VEGF-targeted imaging agent, we coupled anti-VEGF antibody to dextran-coated $\mathrm{Fe}_{3} \mathrm{O}_{4}$ NPs to form anti-VEGF-NPs. Particle size and size distribution of NPs and anti-VEGF-NPs were determined by DLS (Figure 2A). The diameters of NPs and anti-VEGF-NPs were around $49.7 \mathrm{~nm}$ and $57.2 \mathrm{~nm}$, respectively. In addition, the $\mathrm{Fe}_{3} \mathrm{O}_{4}$-based NPs were monodisperse, as shown in the representative TEM images in Figure 2B. Bioconjugation of anti-VEGF antibody to NPs increased the average particle size from $15-30 \mathrm{~nm}$ to $45-65 \mathrm{~nm}$ (Figure 2B). To evaluate the tumor targeting of anti-VEGF-NPs after systemic injection, we performed in vivo imaging on mice bearing colon tumors. On day 14 after CT26 cell inoculation, antiVEGF-NPs or NPs were injected via the jugular vein and T2*-weighted images obtained using a 7T MRI system. As shown in Figure 2C, both the anti-VEGF-NP and NP groups gave a bright signal in the tumor before the administration of nanoparticles. At 1 hour after injection of anti-VEGF-NPs a slight decrease in MRI was noted, as shown by a "blackening signal" in the tumor (data not shown). The anti-VEGF-NP group showed a marked MRI contrast decrease on days 1 and 2 , followed by a slight decline on day 9 . In contrast, the NP group showed a slight decrease on day 1 , with no change on days 2 and 9 when compared to the same animal (controls) before NP injection. There was a $1.52 \pm 0.15,1.54 \pm 0.14$, or $1.34 \pm 0.11$-fold change in the MRI signal within the colon tumor of the mouse on days 1, 2, or 9 after anti-VEGF-NP injection when compared to the control group without nanoparticle injection (Figure 3). In contrast, the signal intensity for the NP-injected group did not show any significant difference from the control value at any time point. The relative contrast intensity change for the anti-VEGF-NP group on days 1 and 2, but not day 9, was significantly increased compared to that in the NP group. These results show that the anti-VEGF-NPs specifically targeted the colon cancer and effectively increased MRI contrast intensity changes.
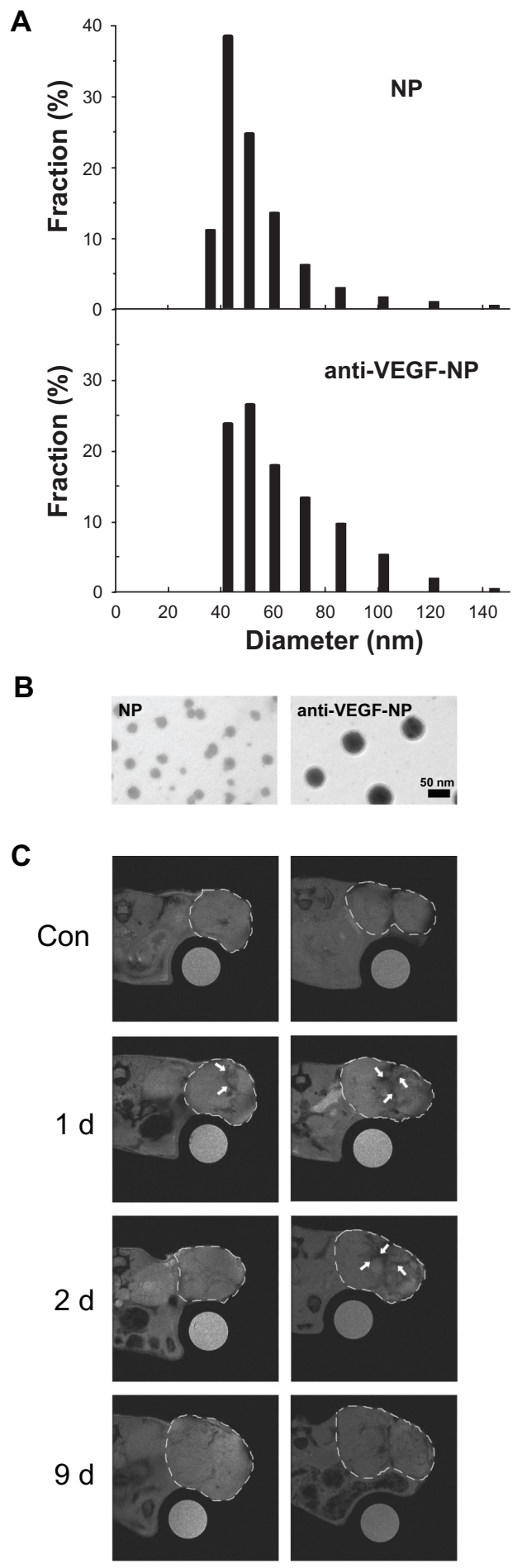

Figure 2 In vivo MRI of anti-VEGF-NPs or NPs injected into mice bearing colon tumors. (A) Particle size and distribution of NPs and anti-VEGF-NPs were determined by DLS method. (B) Representative TEM image of NPs and anti-VEGFNPs; the particle sizes ranged between 15-30 and 45-65 nm, respectively. Scale bar $=50 \mathrm{~nm}$. (C) In vivo MRI of mice bearing colon tumors at the indicated time points ( $1 \mathrm{~d}, 2 \mathrm{~d}$, and $9 \mathrm{~d}$ ) after intravenous injection of $10 \mathrm{mg} \mathrm{Fe} / \mathrm{Kg}$ of NPs (left panels) or anti-VEGF-NPs (right panels).

Notes: The same mice before injection of anti-VEGF-NPs or NPs were used as controls. The dashed lines and arrows indicate the location of the colon tumor and the location of the nanoparticles, respectively.

Abbreviations: MRI, magnetic resonance imaging; VEGNF-NPs, vascular endothelial growth factor nanoparticles; NPs, nanoparticles; DLS, dynamic light scattering; TEM, transmission electron microscopy; d, day. 


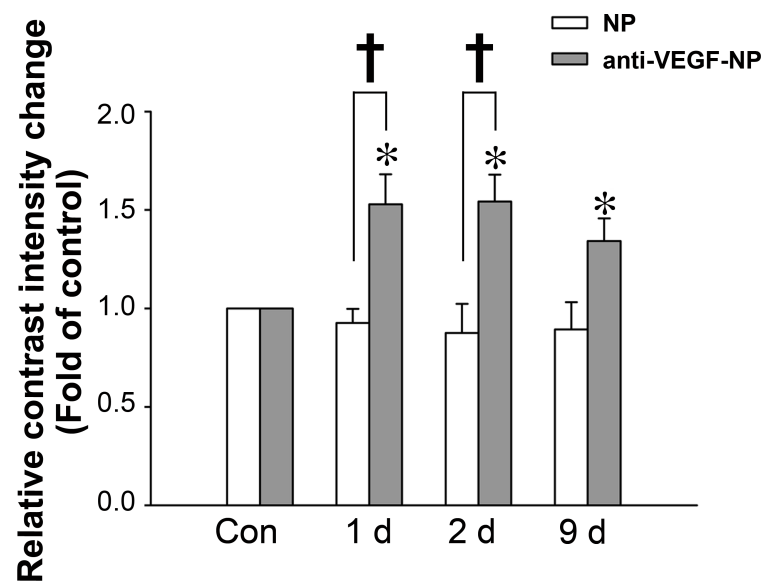

Figure $3 \mathrm{MRI}$ contrast intensity changes from the colon tumor region in vivo at the indicated time in the anti-VEGF-NP and NP groups.

Notes: Using the MRI signal level of the water phantom as a baseline, there is a $1.52 \pm 0.15,1.54 \pm 0.14$, or $1.34 \pm 0.1 \mathrm{I}$-fold change in the MRI signal within the colon tumor of the mouse on day I, 2, or 9 after anti-VEGF-NP injection compared to the control group without nanoparticle injection. The higher level of the relative contrast intensity change suggests that more particles accumulated in the tissue. The data are expressed as the mean \pm SEM for three animals. *Indicates a significant difference compared to the control $(P<0.05)$; ${ }^{\dagger}$ indicates a significant difference between two groups at the indicated time $(P<0.05)$.

Abbreviations: MRI, magnetic resonance imaging; VEGF-NP, vascular endothelial growth factor nanoparticles; NP, nanoparticles; SEM, standard error of the mean; P, probability.

\section{Anti-VEGF-NPs, but not NPs, are specifically retained in the colon cancer mass}

To further explore the distribution of anti-VEGF-NPs and NPs in colon tumors, Prussian blue staining was used to detect iron, representing the location of the nanoparticles. ${ }^{20}$ As shown in Figure 4, Prussian blue staining was seen in cancer sections from mice in the anti-VEGF-NP group on days 1, 2, and 9. The anti-VEGF-NPs were clearly retained in endothelial cells, in the area around blood vessels, and in tumor cells. In contrast, Prussian blue staining was mainly observed in endothelial cells (and in very few tumor cells) among NP-injected mice on day 1; however, no Prussian blue staining was found in the cancer tissues on days 2 and 9. These data show that anti-VEGF-NPs were specifically internalized by endothelial cells and tumor cells as a result of the overexpression of VEGF in tumor tissues.

To determine the biodistribution of nanoparticles in normal tissue in vivo, major organs were harvested, paraffinembedded, sectioned, and stained with Prussian blue on days 1, 2, and 9 after nanoparticle injection. As shown in Figure 5, sections from liver and spleen from both the anti-VEGF-NP and NP group showed blue iron staining, and the quantity of iron deposited was similar on all three days. In contrast, Prussian blue-stained cells were rarely detected in sections from kidney and lung at any time point.

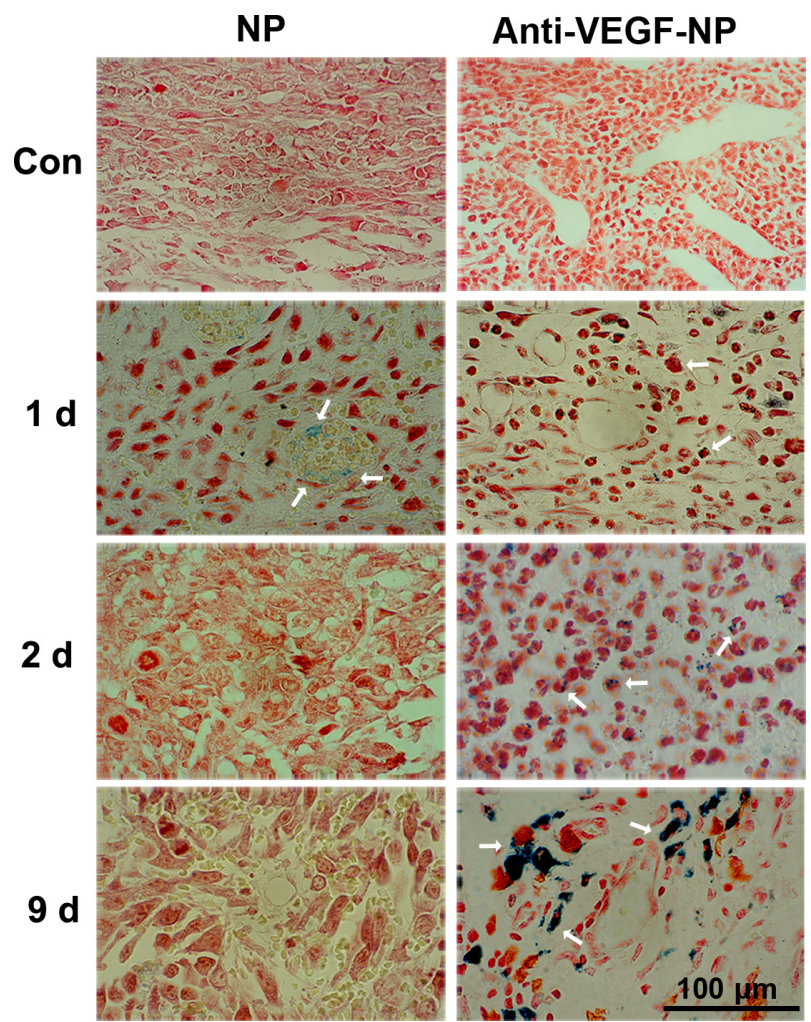

Figure 4 Anti-VEGF-NPs, but not NPs, are specifically retained in the colon cancer mass as shown by Prussian blue staining.

Notes: Tumors were harvested, paraffin-embedded, sectioned, and stained with Prussian blue before anti-VEGF-NP or NP injection (Con) or at day I, 2, or 9 after anti-VEGF-NP or NP injection. The arrows indicate the location of nanoparticles. Scale bar $=100 \mu \mathrm{m}$.

Abbreviations: VEGF-NPs, vascular endothelial growth factor nanoparticles; NPs, nanoparticles; Con, control.

\section{Uptake of anti-VEGF-NPs by tumor cells}

To confirm the distribution of anti-VEGF-NPs and NPs in tumor tissues, TEM was used to visualize the intracellular location of nanoparticles. As shown in Figure 6, tumor sections from anti-VEGF-NP-injected mice on day 1 after injection showed the presence of nanoparticles in the lumen of blood vessels, on the plasma membrane, in the cytoplasm of endothelial cells (Figure 6A), in the extracellular matrix (Figure 6B), and in intracellular granules of tumor cells (Figure 6C). After internalization, the nanoparticles were found to be clumped in lysosomes (Figure 6D). In contrast, only a few NPs were found in tumor cells and in the cytoplasm of endothelial cells (Figure 6E and F). Figure 6G and H show the sections from control non-injected mice.

\section{Discussion}

Colon cancer is a highly aggressive tumor with distinct histopathological features, including high proliferation and necrosis and considerable neovascularization (ie, angiogenesis). ${ }^{21}$ It is generally accepted that the degree of 


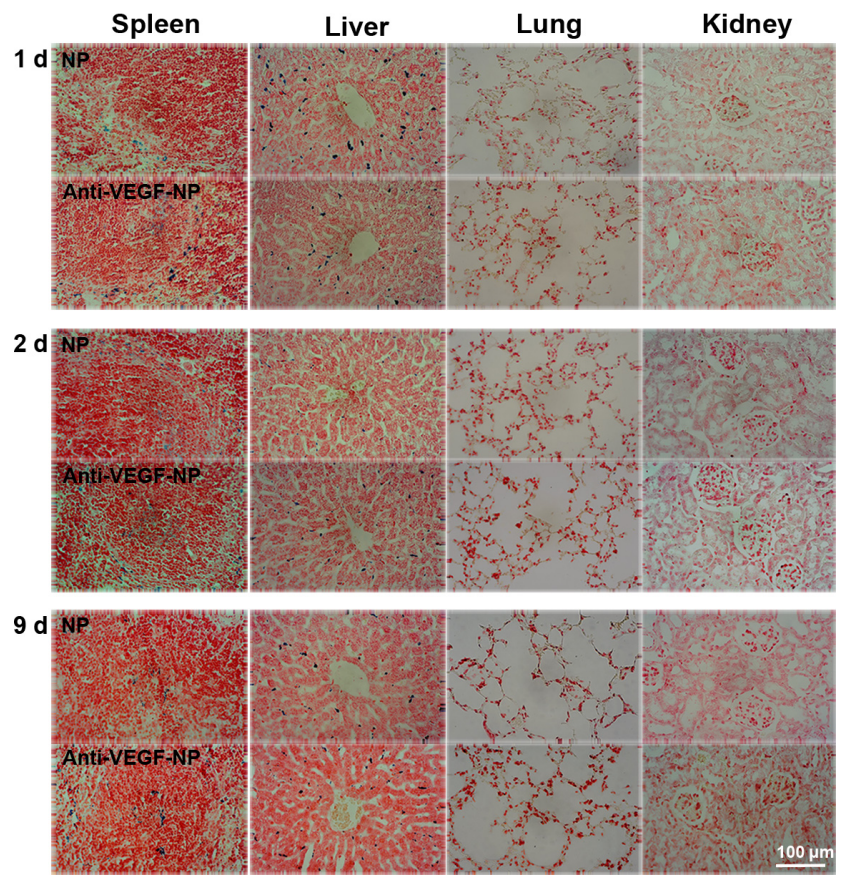

Figure 5 Anti-VEGF-NPs or NPs are present in the spleen and liver, but not the lung and kidney, as shown by Prussian blue staining.

Notes: The spleen, liver, lung, and kidney were harvested from mice bearing colon tumors, paraffin-embedded, sectioned, and stained with Prussian blue staining at day I, 2, or 9 after anti-VEGF-NP or NP injection. Scale bar $=100 \mu \mathrm{m}$.

Abbreviations: VEGF-NPs, vascular endothelial growth factor; NPs, nanoparticles.

angiogenesis correlates with the malignancy of the tumor. ${ }^{22}$ VEGF was chosen for our study, since it is an important mediator in tumor angiogenesis and the pathogenesis of human cancers. ${ }^{13,23}$ In the present study we demonstrated that VEGF was strongly expressed in both human colon tumors and a mouse model of colon tumor, whereas little or no VEGF expression was seen in normal human colon tissue or in other mouse organs by immunohistochemistry. Western blotting confirmed the high expression of VEGF in CT26 cells and colon tumors. The present study demonstrated that VEGF is a selective and abundantly expressed biomarker in Balb/c mice bearing colon tumors that is similar with that in human colon cancer, and that the mice served as a good animal model. Use of an animal model could be exploited for the selective targeting of tumors for imaging and therapeutic applications.

Over the past decade, nanoscience and nanotechnology have provided new concepts for the development of cancer diagnosis by way of imaging or drug target delivery systems; however, challenges for the development of tumor-targeted NPs for in vivo applications have also been recognized. For instance, the poor penetration of drugs across the vascular wall and into the tumor parenchyma against the elevated interstitial pressure in tumors remains a major
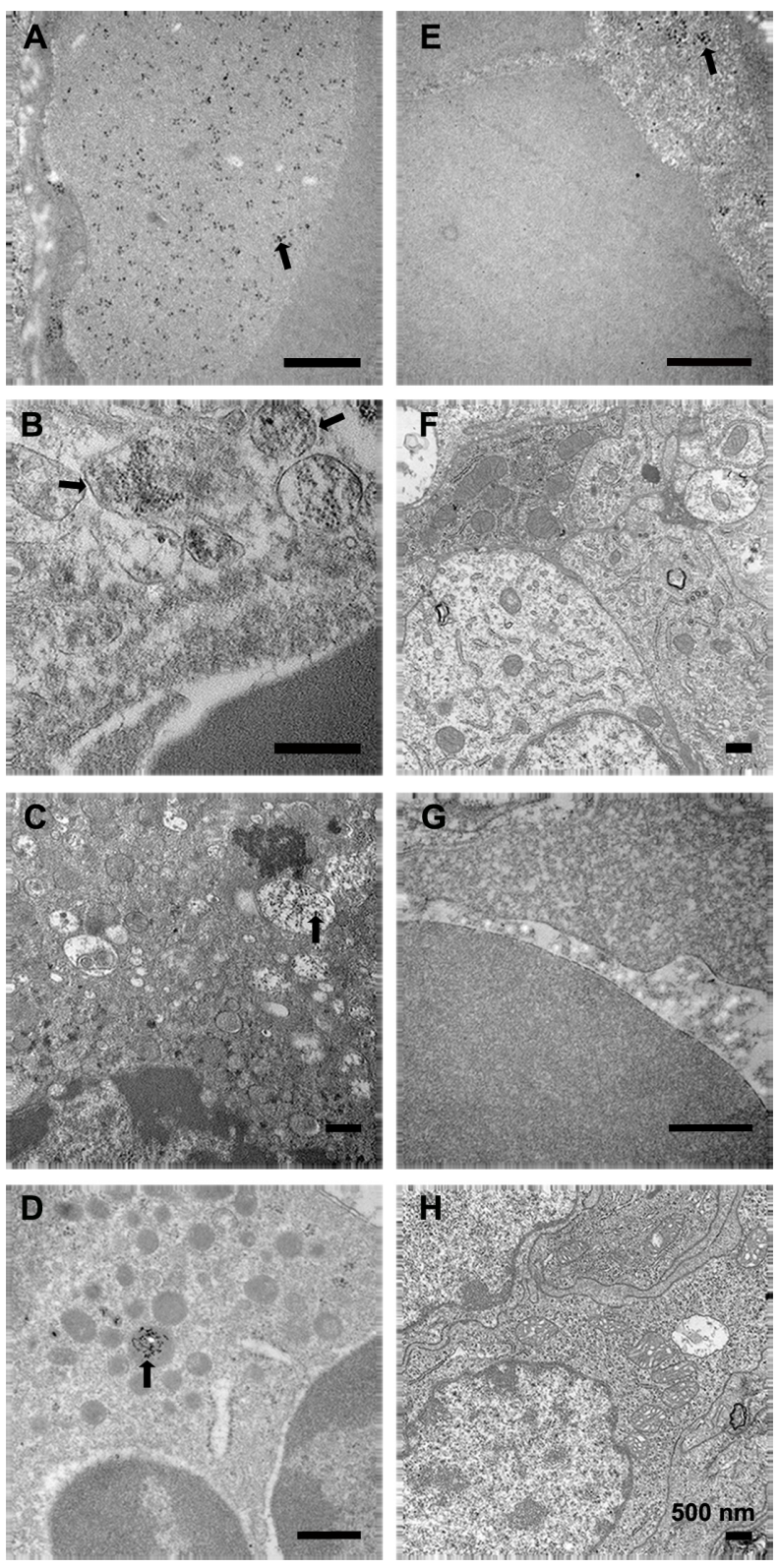

Figure 6 Representative TEM of tumors in mice treated with anti-VEGF-NPs or NPs. On day I after nanoparticle injection, the tumors were fixed with paraformaldehyde, incubated with osmium tetroxide, and embedded in epoxy resin. Thin sections were stained lightly with uranyl acetate and lead citrate to detect uptake of nanoparticles into the cells. (A-D) Anti-VEGF-NPs were present in the vascular lumen (A), endothelial cells and the matrix of surrounding capillaries (B), and tumor cells (C), and were trapped inside lysosomes (D). (E and F) NPs were present in the vascular lumen and the cytoplasm of endothelial cells (E), and seldom found in the extracellular matrix and tumor cells (F). (G and $\mathbf{H}$ ) Tumor sections from mice not injected with nanoparticles. The arrows indicate the location of nanoparticles.

Note: Scale bars $=500 \mathrm{~nm}$.

Abbreviations: TEM, transmission electron micrographs; VEGF-NPs, vascular endothelial growth factor; NPs, nanoparticles.

problem for tumor therapy. ${ }^{24}$ In addition, it is important that nanoparticles not only be stable enough to generate strong imaging signals, but that they also have a modified surface with reactive functional groups for efficient conjugation of tumortargeting ligands and therapeutic agents. Recently, several 
types of NPs, including magnetic iron oxide, quantum dots, gold, and polymer-based NPs, have been developed for cancer applications. ${ }^{25-28}$ Studies have shown that polymer-coated NPs, such as the dextran-coated NPs used in the present study, have minimal impact on cell viability and function, and that they also have low toxicity. ${ }^{18}$ In addition, dextran$\mathrm{Fe}_{3} \mathrm{O}_{4}$ NPs have gained attention as nanocarriers given that they possess several advantages such as their high stability, uniformity of particle size, and high bio-affinity, which make them ideal candidates for the passive targeting of solid tumor tissue sites as a result of the enhanced permeation and retention effect, in which macromolecular agents tend to preferentially accumulate in tumor tissues, and the fact they can be easily and reproducibly prepared in large quantities. ${ }^{29}$ The antibody component attached to the NPs, which allows for the differentiation between specific binding to specific molecular targets, and which allows for nonspecific binding in a 2-4 hour timeframe, may also allow monitoring in a 12-48 hour window, as the antibody may stay bound for a couple of days. ${ }^{20}$

Our previous study demonstrated that anti-VEGF-NPs can be used to explore the relationship between tumor burden and the serum VEGF concentration in rats with hepatocellular carcinoma in an immunomagnetic reduction assay. ${ }^{16}$ In the present study, anti-VEGF-NPs were present in the colon tumor in mice and showed a high signal-to-noise ratio and selective accumulation in the tumor compared to NPs during in vivo optical imaging. The optical imaging statistical data obtained on days 1, 2, and 9 after nanoparticle injection demonstrated a significant change in colon tumor signal in the anti-VEGF-NP-treated group compared to the before injection control group. However, the data showed that a significant change in signal on days 1 and 2 when using anti-VEGF-NPs compared to NPs; however, no differences were noted on day 9. It was hypothesized that NP was cleared and degraded on day 9. This specific issue needs further investigation. Moreover, an added benefit of the anti-VEGFNPs developed in this study is the possibility of using MRI to powerfully and non-invasively assess tumor formation and tumor angiogenesis through high quality anatomic images. After imaging, tissue composition, morphology, and function can be studied in vivo, allowing serial studies to be performed in experimental animals and humans.

The changes in the in vivo MRI signal were correlated with Prussian blue staining. Sections from colon tumorbearing mice injected with anti-VEGF-NPs, but not NPs, showed the presence of nanoparticles in the tumor region that colocalized with the tumor cells and endothelial cells.
Prussian blue-staining confirmed that most of the MRI signal in the colon tumor originated from anti-VEGF-NPs, which was in contrast to the virtual absence of signal using free NPs. Nonspecific biodistribution of anti-VEGF-NPs and NPs was seen in the liver and spleen, but not the kidney and lung, and the amount of iron deposition did not increase with circulation time. These results are consistent with a previous report that NPs can be cleared from the blood and can remain in the liver and spleen, which, as part of the reticuloendothelial system, have phagocytotic activity. ${ }^{30}$

Furthermore, at the end of the imaging protocol on day 1 after injection, tumor sections were analyzed by TEM to examine the localization of the injected anti-VEGF-NPs and NPs. The VEGF-mediated internalization of the targeted nanoparticles into endothelial cells and tumor cells increased the number of nanoparticles inside the tumor mass, whereas NPs were present in the vascular lumen and endothelial cells, but rarely found in tumor cells. These results confirmed that the increased tumor optical signal observed through in vivo optical imaging resulted from the selective 'homing' of anti-VEGF-NPs to abnormal VEGF-expressing vessels, tumor cells, and stroma in tumor tissues, which was consistent with a previous report that endothelial cells, tumor cells, and stroma are important sites of VEGF production in tumor tissues, ${ }^{31}$ making them good molecular targets for VEGF-targeted tumor imaging. Similar results of upregulation of VEGF mRNA expression have been reported for the vast majority of human tumors examined to date, including carcinoma of the lung, breast, gastrointestinal tract, kidney, bladder, and ovary. ${ }^{8-11}$

VEGF is strongly expressed during neoplastic progression. ${ }^{12}$ Based on both reports in the literature and our findings, the strict localization of anti-VEGF-NPs in tumor tissues makes them prime candidates for the detection of other cancers and the development of next-generation agents for targeted therapy. Our results also showed that anti-VEGF-NPs bound to endothelial cells and cancer cells, suggesting that when conjugated to an anticancer drug, they could be used to inhibit the growth of cancer cells and angiogenesis. Moreover, anti-VEGF-NPs -mediated MRI employed in the present study could be combined with multimodal imaging approaches including PET, SPECT, and US, which may lead to improved diagnostic accuracy and simultaneous therapy. ${ }^{32}$

This is the first attempt at detecting in vivo VEGF expression using dextran-coated $\mathrm{F}_{3} \mathrm{O}_{4}$ nanoparticles in conjunction with MRI. Anti-VEGF-NPs showed a significantly higher uptake by tumor tissues than the control NPs. Anti-VEGF- 
NPs are stable, can retain target specificity for VEGFexpressing tumor cells, and enable VEGF-targeted MRI of colon tumors. Our MRI data, combined with light microscopic and TEM detection of the anti-VEGF probe in tumor tissue, provide compelling evidence that this technique can be used to detect VEGF levels in vivo in tumor tissues, and can also be extended to the applications of tissue engineering, which is closely associated with VEGF expression.

\section{Acknowledgments}

The first two authors contributed equally to this study. This work was supported by research grants from the National Science Council (NSC-99-2320-B-002-022-MY3 and NSC96-2314-B-002-150-MY3), the National Taiwan University Hospital (NTUH-099-001310 and NTUH-100-001662), and the Chi-Hua Educational Foundation (QH-2011-001). The MRI studies were supported by the Department of Nuclear Medicine, NTUH and the Small Animal PET/CT Core Facility of Neurobiology and Cognitive Science Center, National Taiwan University.

\section{Disclosure}

The authors report no conflicts of interest in this work.

\section{References}

1. Garcia M, Jemal A, Ward EM, et al. Global Cancer Facts and Figures 2007. Atlanta, GA: American Cancer Society; 2007:1-50.

2. Jemal A, Siegel R, Ward E, et al. Cancer statistics, 2008. CA Cancer J Clin. 2008;58(2):71-96.

3. Wang X, Feng Y, Ke T, Schabel M, Lu ZR. Pharmacokinetics and tissue retention of (Gd-DTPA)-cystamine copolymers, a biodegradable macromolecular magnetic resonance imaging contrast agent. Pharm Res. 2005;22(4):596-602.

4. Peng XH, Qian X, Mao H, et al. Targeted magnetic iron oxide nanoparticles for tumor imaging and therapy. Int $J$ Nanomedicine. 2008;3(3):311-321.

5. Takahashi Y, Kitadai Y, Bucana CD, Cleary KR, Ellis LM. Expression of vascular endothelial growth factor and its receptor, KDR, correlates with vascularity, metastasis, and proliferation of human colon cancer. Cancer Res. 1995;55(18):3964-3968.

6. Ferrara N. Vascular endothelial growth factor: basic science and clinical progress. Endocr Rev. 2004;25(4):581-611.

7. Yamazaki Y, Nakano Y, Imamura T, Morita T. Augmentation of vascular permeability of VEGF is enhanced by KDR-binding proteins. Biochem Biophys Res Commun. 2007;355(3):693-699.

8. Volm M, Koomägi R, Mattern J. Prognostic value of vascular endothelial growth factor and its receptor Flt-1 in squamous cell lung cancer. Int J Cancer. 1997;74(1):64-68.

9. Brown LF, Berse B, Jackman RW, et al. Expression of vascular permeability factor (vascular endothelial growth factor) and its receptors in adenocarcinomas of the gastrointestinal tract. Cancer Res. 1993;53(19):4727-4735.

10. Brown LF, Berse B, Jackman RW, et al. Expression of vascular permeability factor (vascular endothelial growth factor) and its receptors in breast cancer. Hum Pathol. 1995;26(1):86-91.

11. Suzuki K, Hayashi N, Miyamoto Y, et al. Expression of vascular permeability factor/vascular endothelial growth factor in human hepatocellular carcinoma. Cancer Res. 1996;56(13):3004-3009.
12. Guba M, Seeliger H, Kleespies A, Jauch KW, Bruns C. Vascular endothelial growth factor in colorectal cancer. Int $J$ Colorectal Dis. 2004;19(6):510-517.

13. Hicklin DJ, Ellis LM. Role of the vascular endothelial growth factor pathway in tumor growth and angiogenesis. J Clin Oncol. 2005;23(5):1011-1027.

14. Tugues S, Koch S, Gualandi L, Li X, Claesson-Welsh L. Vascular endothelial growth factors and receptors: anti-angiogenic therapy in the treatment of cancer. Mol Aspects Med. 2011;32(2):88-111.

15. Koyama T, Shimura M, Minemoto Y, et al. Evaluation of selective tumor detection by clinical magnetic resonance imaging using antibody-conjugated superparamagnetic iron oxide. J Control Release. 2012;159(3):413-418.

16. Huang KW, Yang SY, Yu CY, et al. Exploration of the relationship between the tumor burden and the concentration of vascular endothelial growth factor in liver-cancer-bearing animals using immunomagnetic reduction assay. J Biomed Nanotechnol. 2011;7(4):535-541.

17. Enochs WS, Harsh G, Hochberg F, Weissleder R. Improved delineation of human brain tumors on MR images using a long-circulating, superparamagnetic iron oxide agent. J Magn Reson Imaging. 1999;9(2):228-232.

18. Thorek DL, Chen AK, Czupryna J, Tsourkas A. Superparamagnetic iron oxide nanoparticle probes for molecular imaging. Ann Biomed Eng. 2006;34(1):23-38.

19. Chen YH, Lin SJ, Chen JW, Ku HH, Chen YL. Magnolol attenuates VCAM-1 expression in vitro in TNF-alpha-treated human aortic endothelial cells and in vivo in the aorta of cholesterol-fed rabbits. Br J Pharmacol. 2002;135(1):37-47.

20. Towner RA, Smith N, Asano Y, et al. Molecular magnetic resonance imaging approaches used to aid in the understanding of angiogenesis in vivo: implications for tissue engineering. Tissue Eng Part A. 2010;16(2):357-364.

21. Köhne CH, Lenz HJ. Chemotherapy with targeted agents for the treatment of metastatic colorectal cancer. Oncologist. 2009;14(5):478-488.

22. Daumas-Duport C, Varlet P, Tucker ML, Beuvon F, Cervera P, Chodkiewicz JP. Oligodendrogliomas. Part I: Patterns of growth, histological diagnosis, clinical and imaging correlations: a study of 153 cases. J Neurooncol. 1997;34(1):37-59.

23. Grothey A, Galanis E. Targeting angiogenesis: progress with anti-VEGF treatment with large molecules. Nat Rev Clin Oncol. 2009;6(9):507-518.

24. Sugahara KN, Teesalu T, Karmali PP, et al. Tissue-penetrating delivery of compounds and nanoparticles into tumors. Cancer Cell. 2009;16(6):510-520.

25. Yang L, Mao H, Wang YA, et al. Single chain epidermal growth factor receptor antibody conjugated nanoparticles for in vivo tumor targeting and imaging. Small. 2009;5(2):235-243.

26. Cai W, Shin DW, Chen K, et al. Peptide-labeled near-infrared quantum dots for imaging tumor vasculature in living subjects. Nano Lett. 2006;6(4):669-676.

27. Lee JH, Huh YM, Jun YW, et al. Artificially engineered magnetic nanoparticles for ultra-sensitive molecular imaging. Nat Med. 2007;13(1):95-99.

28. Nasongkla N, Bey E, Ren J, et al. Multifunctional polymeric micelles as cancer-targeted, MRI-ultrasensitive drug delivery systems. Nano Lett. 2006;6(11):2427-2430.

29. Jiang WQ, Yang HC, Yang SY, et al. Preparation and properties of superparamagnetic nanoparticles with narrow size distribution and biocompatible. J Magn Magn Mater. 2004;283(2-3):210-214.

30. Almeida JP, Chen AL, Foster A, et al. In vivo biodistribution of nanoparticles. Nanomedicine (Lond). 2011;6(5):815-835.

31. Fukumura D, Xavier R, Sugiura T, et al. Tumor induction of VEGF promoter activity in stromal cells. Cell. 1998;94(6):715-725.

32. Malvindi MA, Greco A, Conversano F, et al. Magnetic/silica nanocomposites as dual-mode contrast agents for combined magnetic resonance imaging and ultrasonography. Adv Funct Mater. 2011;21(13): $2548-2555$. 


\section{Publish your work in this journal}

The International Journal of Nanomedicine is an international, peerreviewed journal focusing on the application of nanotechnology in diagnostics, therapeutics, and drug delivery systems throughout the biomedical field. This journal is indexed on PubMed Central, MedLine, CAS, SciSearch $\AA$, Current Contents ${ }^{\circledR} /$ Clinical Medicine,

Journal Citation Reports/Science Edition, EMBase, Scopus and the Elsevier Bibliographic databases. The manuscript management system is completely online and includes a very quick and fair peer-review system, which is all easy to use. Visit http://www.dovepress.com/ testimonials.php to read real quotes from published authors.

Submit your manuscript here: http://www.dovepress.com/international-journal-of-nanomedicine-journal 